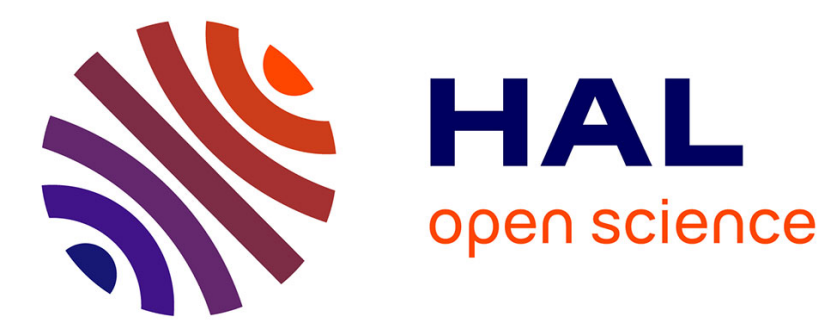

\title{
Sliding mode observer for proton exchange membrane fuel cell: automotive application
}

Maxime Piffard, Mathias Gerard, Ramon da Fonseca, Paolo Massioni, Eric Bideaux

\section{- To cite this version:}

Maxime Piffard, Mathias Gerard, Ramon da Fonseca, Paolo Massioni, Eric Bideaux. Sliding mode observer for proton exchange membrane fuel cell: automotive application. Journal of Power Sources, 2018, 338, pp.71-77. 10.1016/j.jpowsour.2018.03.057 . hal-01740933

\section{HAL Id: hal-01740933 \\ https://hal.science/hal-01740933}

Submitted on 30 Apr 2019

HAL is a multi-disciplinary open access archive for the deposit and dissemination of scientific research documents, whether they are published or not. The documents may come from teaching and research institutions in France or abroad, or from public or private research centers.
L'archive ouverte pluridisciplinaire HAL, est destinée au dépôt et à la diffusion de documents scientifiques de niveau recherche, publiés ou non, émanant des établissements d'enseignement et de recherche français ou étrangers, des laboratoires publics ou privés. 


\title{
Sliding mode observer for proton exchange membrane fuel cell: automotive application
}

\author{
Maxime Piffard ${ }^{\mathrm{a}, \mathrm{b}}$, Mathias Gerard ${ }^{\mathrm{a}, \mathrm{b}}$, Ramon Da Fonseca ${ }^{\mathrm{a}, \mathrm{b}}$, Paolo \\ Massioni $^{\mathrm{c}}$, Eric Bideaux ${ }^{\mathrm{c}}$ \\ ${ }^{a}$ CEA LITEN, F-38054 Grenoble, France (e-mail: maxime.piffard@cea.fr) \\ ${ }^{b}$ Univ. Grenoble Alpes, F-38000 Grenoble, France \\ ${ }^{c}$ Laboratoire Ampère, INSA Lyon, Université de Lyon, 69621 Villeurbanne CEDEX, France
}

\begin{abstract}
This work proposes a state observer as a tool to manage cost and durability issues for PEMFC (Proton Exchange Membrane Fuel Cell) in automotive applications. Based on a dead-end anode architecture, the observer estimates the nitrogen build-up in the anode side, as well as relative humidities in the channels. These estimated parameters can then be used at fuel cell management level to enhance the durability of the stack. This observer is based on transport equations through the membrane and it reconstructs the behavior of the water and nitrogen inside the channels without the need of additional humidity sensors to correct the estimate. The convergence of the output variables is proved with Lyapunov theory for dynamic operating conditions. The validation is made with a high-fidelity model running a WLTC (Worldwide harmonized Light vehicles Test Cycle). This observer provide the average values of nitrogen and relative humidities with sufficient precision to be used in a global real-time control scheme.
\end{abstract}

Keywords:

PEMFC, fuel cell management, state observer, sliding mode observer

\section{Introduction}

Proton Exchange Membrane Fuel Cell (PEMFC) seems to be the most suitable Fuel Cell (FC) technology for transportation applications, thanks to the 
high power density and the low operating temperature [1]. The remaining challenges to overcome before the widespread commercialization are the cost, durability and start-up, according to [2]. They can be improved either by materials improvements or by system optimization. For example, the dead-end anode (DEA) is a simple solution to run a fuel cell system with a reduced number of ancillaries. This also improves the fuel utilization as the hydrogen injected in the anode is supposed to be fully consumed, leading to a hydrogen efficiency close to $100 \%$. However, the main drawback of the DEA is the presence of permeated nitrogen and liquid water in the anode side, that can only be removed of the channels by the outlet valve.

Several studies have analysed the issue of nitrogen and liquid water build up in the anode channels. According to [3], these species are responsible of a voltage drop as the stratification phenomenon tends to lower the hydrogen concentration in the outlet on the channels. This voltage drop is reversible, as the full voltage is recovered if the nitrogen and liquid water are purged from the anode. However, their presence could also lead to irreversible degradations, in particular the cathode carbon corrosion: the link between the carbon corrosion and the nitrogen build up in the anode has been studied by [4] during long purge intervals. Therefore, the DEA architecture is a solution to the cost issue but there is a need of an optimized purge procedure to minimize reversible and irreversible degradations from nitrogen build up.

The purge optimization has been studied in steady-state operations. For example, [5] reports a trade-off between long purge intervals (responsible of carbon corrosion) and short ones (where the hydrogen loss is increased). A solution is given by taking into account the opening and closing time constants of the actuators. The optimization of the purge interval and duration is performed for a given operating power. However, this optimization is dependent of the operating conditions and it can not be applied to dynamic power. A second optimization has been made by taking into account the relative humidity in the channels in [6]. Another optimization purge has been performed for a $90 \mathrm{~kW}$ automotive stack in recirculation mode by $[7]$ to minimize the performance drop. 
In this case, the impact of nitrogen permeation on the performance is lower as the nitrogen is diluted in the hydrogen instead of accumulating in the outlet. The main drawback of these studies is that they can not deal with dynamic power and/or varying operating conditions. Adaptation to dynamic power is mandatory for automotive applications and varying operating conditions rather than steady-state conditions seems to be the next step of FC control. The publications $[8,9,10]$ are some examples of the potential of dynamic operating conditions.

The design of such advanced FC control often requires the use of humidity measurements inside the stack. However, humidity sensors are expensive, have a poor durability and reliability and can only measure the relative humidity outside the stack, in the subsystems. A solution could be to build a diagnosis tool for flooding and drying of the cell based on a database, as in [11]. Another solution is to build a state observer to estimate the relative humidities in the channels, as in [12], where they estimate precisely the distribution of state parameters along the channels but rely on humidity sensors to correct the estimation.

The work presented here is based on a DEA architecture and proposes an observer to estimate the nitrogen in the anode side and the relative humidities in the channels. Only four types of sensors are used to correct the estimation: pressure, temperature, voltage and current. The objective is to provide a simple tool to generate additional measurements for a fuel cell management system. The observer is based on a simplified model which approximates a complex " $2 \mathrm{D}+1 \mathrm{D} "$ model (more details are given in the next section). The observer convergence is proved for dynamic operating conditions with Lyapunov theory. The estimation precision is validated using the complex " $2 \mathrm{D}+1 \mathrm{D}$ " model with the desired output power extracted from WLTC profile.

A previous work with the same purpose has been presented in [13] with the UKF technique (Unscented Kalman Filter) for the observer instead of sliding mode. This UKF observer is difficult to tune for a specific $(P, T)$ couple with no theoretical proof of convergence. The proposed observer is based on a more so- 
phisticated state-space model and a different algorithm to overcome those issues. The extensive validation framework, using a wide range of dynamical operating conditions, disturbances and durability concerns, shows the effectiveness of this new approach versus [13].

\section{Nomenclature}

$\lambda_{i} \quad$ Membrane water content at the interface with $i$

$\lambda_{m} \quad$ Membrane water content

$\rho_{d r y} \quad$ Dry membrane density

$\left[\mathrm{kg} \cdot \mathrm{m}^{3}\right]$

$e_{m} \quad$ Membrane thickness

$[\mathrm{m}]$

$E W \quad$ Equivalent weight of the membrane

F $\quad$ Faraday constant

$\left[\mathrm{C} \cdot \mathrm{mol}^{-1}\right]$

$F_{d, i} \quad$ Back-diffusion flux of water

$\left[\mathrm{mol} \cdot \mathrm{s}^{-1}\right]$

$F_{e o} \quad$ Electro-osmosis drag

$\left[\mathrm{mol} \cdot \mathrm{s}^{-1}\right]$

$F_{i n, H_{2}}$ Inlet hydrogen flux in the anode

$\left[\mathrm{mol} \cdot \mathrm{s}^{-1}\right]$

$F_{\text {in }} \quad$ Inlet flux of water in the cathode

$\left[\mathrm{mol} \cdot \mathrm{s}^{-1}\right]$

$F_{\text {out }}$ Outlet flux of water in the cathode

$\left[\mathrm{mol} \cdot \mathrm{s}^{-1}\right]$

$F_{X, p} \quad$ Outlet flux of specie $X$ during the purge

$\left[\mathrm{mol} \cdot \mathrm{s}^{-1}\right]$

I Stack current

$i \quad a$ for anode, $c$ for cathode

$K_{N_{2}} \quad$ Nitrogen permeation flux

$\left[\mathrm{mol} \cdot \mathrm{s}^{-1}\right]$

$n_{X, i} \quad$ Moles of specie $X$ in the channel $i$

[mol]

$P_{i} \quad$ Pressure in the channel i 
$P_{\text {sat }} \quad$ Saturation vapour pressure

$P_{v a p} \quad$ Partial vapour pressure

$Q_{a o} \quad$ Purge command

$R \quad$ Perfect gas constant

$\left[\mathrm{J} \cdot \mathrm{K}^{-1} \cdot \mathrm{mol}^{-1}\right]$

$R_{m} \quad$ Membrane resistance

$S \quad$ Membrane surface

$T \quad$ Stack temperature

$V_{i} \quad$ Volume of the channel $i$

$\left[\mathrm{m}^{3}\right]$

$X_{X, i} \quad$ Molar fraction of specie $X$ in the channel $i$

\section{Fuel Cell model}

\subsection{General description}

The fuel cell model used in this work is a model called MePHYSTO-FC, developed since several years and described in many studies $[14,15,16]$ (previously called PS++ model).

It is a development platform included a $2 \mathrm{D}+1 \mathrm{D}$ multi-physic fuel cell model based on lumped and bond graph approach. It takes into account gas diffusion, two phases flow, heat transfer and electrochemistry. The complex in-plane serpentine flow field of the bipolar plates is modeled in 2D. The through-plane species transports are modeled in 1D (no in-plane transport in the GDL and catalyst layer). The model is used to calculate the local conditions and current distribution over the surface of the cell in response to dynamic operating conditions. Degradation mechanisms are added (by bottom-up of top-down approach) to calculate the fuel cell lifetime. A in-depth description of the actual model can be found in [16]. 


\subsection{Membrane model}

We recall here the main equations involved in the membrane transport species in our model. These equations are used in the observer. All the symbols are defined in the nomenclature.

The membrane water content $\lambda_{m}$ is estimated with an integral term as:

$$
\begin{aligned}
& \lambda_{m}=\int\left(\frac{E W}{\rho_{d r y}} \frac{1}{e_{m} S}\left(-\left(F_{d, a}-F_{e o}\right)-\left(F_{d, c}+F_{e o}\right)\right)\right) d t \\
& \lambda_{m}=\int\left(\frac{E W}{\rho_{d r y}} \frac{1}{e_{m} S}\left(-F_{d, a}-F_{d, c}\right)\right) d t
\end{aligned}
$$

The electro-osmosis and back-diffusion fluxes are calculated with [14]:

$$
\begin{aligned}
F_{e o}= & \left(1.0+0.028 \lambda_{m}+0.0026 \lambda_{m}^{2}\right) \frac{I}{F} \\
F_{d, i}= & \frac{\rho_{d r y}}{E W} \frac{S}{e_{m}}\left(6.707 \cdot 10^{-8} \lambda_{m}+6.387 \cdot 10^{-7}\right) \\
& \cdot \exp \left(\frac{-2416}{T}\right)\left(\lambda_{m}-\lambda_{i}\right)
\end{aligned}
$$

with $\lambda_{i}$ the membrane water content at the interface between the membrane and the electrode active area given by:

$$
\lambda_{i}=0.043+17.81 a-39.85 a^{2}+36 a^{3}
$$

where $a=P_{\text {vap }} / P_{\text {sat }}(T)$.

The model for the nitrogen permeation is taken from [4]:

$$
\begin{aligned}
& K_{N_{2}}\left(\lambda_{m}, T\right)=\alpha_{N_{2}}\left(0.0295+1.21 f_{v}-1.93 f_{v}^{2}\right) \cdot 10^{-14} \\
& . \exp \left[\frac{E_{N_{2}}}{R}\left(\frac{1}{T_{\text {ref }}}-\frac{1}{T}\right)\right]
\end{aligned}
$$

with $\alpha_{N_{2}}$ a scale factor, $E_{N_{2}}$ the nitrogen molar energy $\left(E_{N_{2}}=24 \mathrm{kJmol}^{-1}\right)$, $T_{r e f}=303 \mathrm{~K}$ and $f_{v}$ the volume fraction of water in the membrane, given by:

$$
f_{v}=\frac{\lambda_{m} V_{w}}{V_{m b}+\lambda_{m} V_{w}}
$$


with $V_{w}$ the molar volume of water and $V_{m b}=E W / \rho_{d r y}$ the dry membrane volume.

\subsection{System model}

Despite the complex model of the fuel cell stack, the system is considered perfect for observation purposes. We assume that the air pressure is appropriatly regulated, as well as the inlet air flow. The cooling temperature enter the stack at a constant value. The inlet cathode humidity is also fixed at a given value and the anode is fed with dry hydrogen.

The hydrogen sub-system model is more detailed to take into account deadend phenomena. A model of a hydrogen vessel and a model of pressure regulator are included at the inlet of the stack, as well as a dead volume and a solenoid valve at the outlet.

\section{State observer}

\subsection{Assumptions}

The proposed work is based on the following assumptions.

i/ homogeneous phenomena inside the cell. It allows considering a unique volume, pressure and temperature in the channels. As a consequence, the dynamical model of the observer does not have partial derivative equations. This results in a reduced computational time.

ii/ absence of liquid water in the channels. The observer model consider that the entire amount of water is in the vapour phase. The build up of liquid water is still detected but badly estimated.

iii/ the membrane water content dynamics is faster than the temperature dynamics. This condition can be written as:

$$
\frac{\dot{\lambda}_{m}}{\lambda_{m}} \gg \frac{\dot{T}}{T}
$$




$$
\left\{\begin{aligned}
\dot{n}_{N_{2}, a}(t) & =K_{N_{2}}\left(\lambda_{m}(t), T\right)\left(X_{N_{2}, a i r} P_{c}-\frac{n_{N_{2}, a}(t) R T}{V_{a}}\right)-F_{N_{2}, p} Q_{a o} \\
\dot{n}_{H_{2} O, a}(t) & =F_{d, a}\left(\lambda_{m}(t), n_{H_{2} O, a}(t), T\right)-F_{e o}\left(\lambda_{m}(t), I\right)-F_{H_{2} O, p} Q_{a o} \\
\dot{n}_{H_{2} O, c}(t) & =F_{d, c}\left(\lambda_{m}(t), n_{H_{2} O, c}(t), T\right)+F_{e o}\left(\lambda_{m}(t), I\right)+\frac{I}{2 F} \\
& +F_{\text {in }}\left(H r_{c, i n}, S t_{O_{2}}, I, T, P_{c}\right)-F_{o u t}\left(n_{H_{2} O, c}(t), S t_{O_{2}}, I, T, P_{c}\right) \\
\dot{\lambda}_{m}(t) & =\frac{E W}{\rho_{d r y} \cdot e_{m} \cdot S}\left[-F_{d, a}\left(\lambda_{m}(t), n_{H_{2} O, a}(t), T\right)-F_{d, c}\left(\lambda_{m}(t), n_{H_{2} O, c}(t), T\right)\right] \\
\dot{n}_{H_{2}, a}(t) & =F_{\text {in, } H_{2}}\left(n_{H_{2}, a}(t), n_{N_{2}, a}(t), n_{H_{2} O, a}(t), T\right)-\frac{I}{2 F}-F_{H_{2}, p} Q_{a o}
\end{aligned}\right.
$$

\subsection{Structure}

The state observer is derived from the 2D fuel cell model described above. The chosen states are the quantity of water in the channels (anode and cathode) and the nitrogen buildup in the anode side. Two additional states are used to compute the dynamical equations and guarantee the observability of the system: the membrane water content and the quantity of hydrogen in the anode side. Therefore, we have the following state vector:

$$
X=\left[\begin{array}{c}
n_{N_{2}, a} \\
n_{H_{2} O, a} \\
n_{H_{2} O, c} \\
\lambda_{m} \\
n_{H_{2}, a}
\end{array}\right]
$$

where $n_{N_{2}, a}$ is the number of moles of nitrogen in the anode, $n_{H_{2} O, a}$ and $n_{H_{2} O, c}$ respectively the number of moles of water, liquid and vapor, in the anode and cathode side, $\lambda_{m}$ the membrane water content and $n_{H_{2}, a}$ the number of moles of hydrogen in the anode.

Based on this state vector and the 2D model, the dynamical equations of the observer are listed in (9).

The input vector considered here is the boolean purge command $Q_{a o}$, the current $I$, the cooling temperature $T$ (measured at the stack cooling outlet) and the cathodic pressure $P_{c}$. Consequently we have: 


$$
U=\left[\begin{array}{c}
Q_{a o} \\
I \\
T \\
P_{c}
\end{array}\right]
$$

The choice of measurements used to correct the states is made as simple as possible. The observer should be able to run on a simple and reliable system, so one should avoid using relative humidity sensors which are expensive. The only two measurements used in the algorithm are the membrane ohmic resistance $R_{m}$ and the anodic pressure $P_{a}$. The membrane ohmic resistance can be obtained with several methods described and compared in [17] ; all of these methods provide at least $3.5 \%$ accuracy. It is assumed in this study that such measurement is available on a real system with a precision up to $3 \%$.

The measurement vector is:

$$
Y=\left[\begin{array}{c}
R_{m} \\
P_{a}
\end{array}\right]
$$

with the membrane ohmic resistance linked to the membrane water content by:

$$
R_{m}=\frac{e_{m}}{S} \frac{1}{33.75 \lambda_{m}-21.41} \exp \left(\frac{1268}{T}\right)
$$

and the anodic pressure expressed with the perfect gas law:

$$
P_{a}=\frac{R T}{V_{a}}\left(n_{H_{2}, a}+n_{H_{2} O, a}+n_{N_{2}, a}\right)
$$

The observer scheme is given in Figure 1.

\subsection{Observability}

The observability of a nonlinear system can be checked with the use of Lie derivatives [18].

Let consider a classical nonlinear system:

$$
\left\{\begin{array}{l}
\dot{x}=f(x, u) \\
y=h(x)=\left[\begin{array}{lll}
h_{1}(x) & \ldots h_{m}(x)
\end{array}\right]^{T}
\end{array}\right.
$$




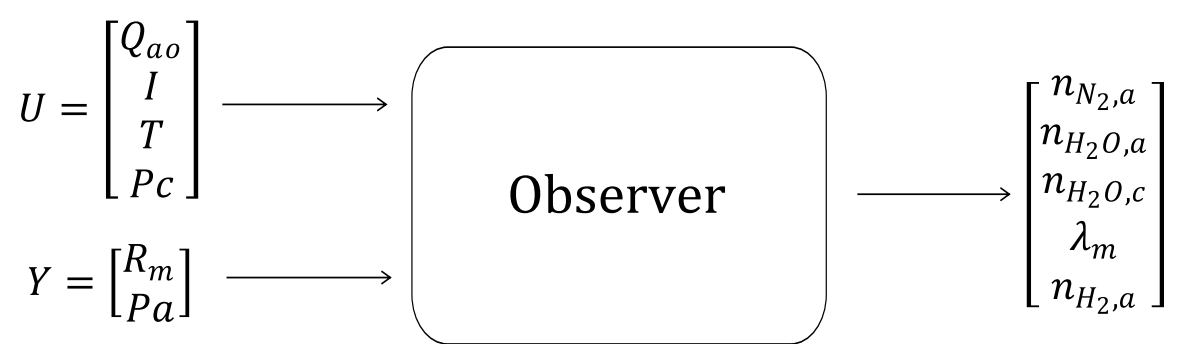

Figure 1: Observer scheme

with $x \in \mathbb{R}^{n}, u \in \mathbb{R}^{l}, y \in \mathbb{R}^{m}$.

Let define the Lie derivative of $h$ with respect to $f$ :

$$
L_{f}(h)=\nabla h . f=\frac{\partial h}{\partial x} f=\sum_{i=1}^{m} \frac{\partial h}{\partial x_{i}} f_{i}
$$

with

$$
L_{f}^{0}(h)=h
$$

and

$$
L_{f}^{(k)}(h)=\frac{\partial}{\partial x}\left[L_{f}^{(k-1)}(h)\right] f
$$

for $k \geq 1$.

One then define the observability matrix based on the Lie derivatives:

$$
O=\left[\begin{array}{ccc}
d L_{f}^{0}\left(h_{1}\right) & \ldots & d L_{f}^{0}\left(h_{m}\right) \\
\ldots & \ldots & \ldots \\
d L_{f}^{n-1}\left(h_{1}\right) & \ldots & d L_{f}^{n-1}\left(h_{m}\right)
\end{array}\right]
$$

The system is said to be locally observable if $O$ is of rank $n$. The observability matrix of the observer mentioned above is algebraically of rank $n$ so we can conclude that the observer is locally observable everywhere. One should notice that it is not possible to conclude to a global observability with this method. 


\subsection{Sliding mode surface and convergence}

The principle of the sliding mode technique is to create a discontinuous control that forces the states to converge to a surface and then stay on it ("slide"). Therefore the definition of the sliding surface is the key parameter of a sliding mode observer or control. Among more technical formulations, a simple and classical solution for a sliding surface is presented here:

$$
\begin{aligned}
\epsilon_{1} & =y_{1}-\hat{y}_{1} \\
\epsilon_{2} & =y_{2}-\hat{y}_{2}
\end{aligned}
$$

where $\epsilon_{1}$ and $\epsilon_{2}$ the sliding surfaces, $y_{1}$ and $y_{2}$ the components of the measurement vector $Y$ and $\hat{y}_{1}, \hat{y}_{2}$ the corresponding estimates by the observer.

The dynamical equations of the sliding mode observer can be written as:

$$
\left\{\begin{array}{l}
\dot{\hat{x}}_{1}=f_{1}(\hat{x}, u)-\sigma_{1} \operatorname{sign}\left(\epsilon_{2}\right) \\
\dot{\hat{x}}_{2}=f_{2}(\hat{x}, u)-\sigma_{2} \operatorname{sign}\left(\epsilon_{2}\right) \\
\dot{\hat{x}}_{3}=f_{3}(\hat{x}, u) \\
\dot{\hat{x}}_{4}=f_{4}(\hat{x}, u)-\sigma_{4} \operatorname{sign}\left(\epsilon_{1}\right) \\
\dot{\hat{x}}_{5}=f_{5}(\hat{x}, u)-\sigma_{5} \operatorname{sign}\left(\epsilon_{2}\right)
\end{array}\right.
$$

The states 1,2 and 5, respectively $n_{N_{2}, a}, n_{H_{2} O, a}$ and $n_{H_{2}, a}$ are corrected with $\epsilon_{2}$ as they appear in the equation of $P_{a}$ in (13). Similarly, the state $4, \lambda_{m}$ is corrected with $\epsilon_{1}$. The last one, $x_{3}$, corresponding to $n_{H_{2} O, c}$, is linked to none of the measurements so it is not corrected directly.

The use of such a sliding surface only guarantees a convergence of the outputs of the observer and not a convergence of the states to the real ones.

The convergence proof of the sliding mode observer is made with the Lyapunov theory, given the two Lyapunov functions:

$$
V_{1}=\frac{1}{2}\left(\epsilon_{1}\right)^{2}
$$


and

$$
V_{2}=\frac{1}{2}\left(\epsilon_{2}\right)^{2}
$$

Deriving these expressions leads to

$$
\dot{V}_{1}=\epsilon_{1}\left(\dot{y}_{1}-\frac{d h_{1}(\hat{x})}{d \hat{x}_{4}} f_{4}(\hat{x}, u)+\frac{d h_{1}(\hat{x})}{d \hat{x}_{4}} \sigma_{4} \operatorname{sign}\left(\epsilon_{1}\right)\right)
$$

and

$$
\dot{V}_{2}=\epsilon_{2}\left(\dot{y}_{2}-\frac{R T}{V_{a}} \sum_{1,2,5} f_{i}(\hat{x}, u)+\frac{R T}{V_{a}} \sum_{1,2,5} \sigma_{i} \operatorname{sign}\left(\epsilon_{2}\right)\right)
$$

The gains must assure $\dot{V}_{1}<0$ and $\dot{V}_{2}<0$ to make that $\epsilon_{1} \rightarrow 0$ and $\epsilon_{2} \rightarrow 0$. Therefore:

$$
\sigma_{4}>\max \left|\frac{\dot{y}_{1}}{\frac{d h_{1}(\hat{x})}{d \hat{x}_{4}}}-f_{4}(\hat{x}, u)\right|
$$

and

$$
\sum_{1,2,5} \sigma_{i}>\max \left|\frac{\dot{y}_{2}}{\frac{R T}{V_{a}}}-\sum_{1,2,5} f_{k}(\hat{x}, u)\right|
$$

The second inequality is a constraint on a sum of gains that gives theoretically infinite possibilities of tuning parameters for a same threshold, as long as the inequality holds. For this study, $\sigma_{2}$ is chosen equal to zero and $\sigma_{1}=\sigma_{5}$ to simplify the experimental tuning. One should notice that by choosing $\sigma_{2}=0$, none of the states relative to the quantity of water in the channels are directly corrected (states 2 and 3 , respectively $n_{\mathrm{H}_{2} \mathrm{O}, a}$ and $n_{\mathrm{H}_{2} \mathrm{O}, \mathrm{c}}$ ).

These inequalities can be modified to ensure the convergence of the observer in finite time by using the condition $\dot{V}<\mu V$ instead of $\dot{V}<0$. One can also ensure insensitivity to model errors by adding bounded uncertain terms $\xi_{i}$ in system (21) to obtain: 


$$
\left\{\begin{array}{l}
\dot{\hat{x}}_{1}=f_{1}(\hat{x}, u)+\xi_{1}-\sigma_{1} \operatorname{sign}\left(\epsilon_{2}\right) \\
\dot{\hat{x}}_{2}=f_{2}(\hat{x}, u)+\xi_{2}-\sigma_{2} \operatorname{sign}\left(\epsilon_{2}\right) \\
\dot{\hat{x}}_{3}=f_{3}(\hat{x}, u)+\xi_{3} \\
\dot{\hat{x}}_{4}=f_{4}(\hat{x}, u)+\xi_{4}-\sigma_{4} \operatorname{sign}\left(\epsilon_{1}\right) \\
\dot{\hat{x}}_{5}=f_{5}(\hat{x}, u)+\xi_{5}-\sigma_{5} \operatorname{sign}\left(\epsilon_{2}\right)
\end{array}\right.
$$

The inequalities (26) and (27) are then modified into:

$$
\begin{gathered}
\sigma_{4}>\max \left|\frac{\dot{y}_{1}}{\frac{d h_{1}(\hat{x})}{d \hat{x}_{4}}}-f_{4}(\hat{x}, u)-\xi_{4}-\frac{\epsilon_{1} \mu_{1}}{2 \frac{d h_{1}(\hat{x})}{d \hat{x}_{4}}}\right| \\
\sum_{1,2,5} \sigma_{i}>\max \left|\frac{\dot{y}_{2}}{\frac{R T}{V_{a}}}-\sum_{1,2,5}\left(f_{k}(\hat{x}, u)+\xi_{k}\right)-\frac{\epsilon_{2} \mu_{2}}{2 \frac{R T}{V_{a}}}\right|
\end{gathered}
$$

By choosing $\sigma_{i}$ sufficiently large, it is therefore possible to get a convergence in finite time, linked to the $\mu$ value, and insensitivity to model errors.

\section{Results \& discussion}

The proposed observer has been validated using the MePHYSTO-FC code running in $1 \mathrm{D}+1 \mathrm{D}$ (1D from inlet to outlet and 1D through the MEA - Membrane Electrode Assembly). The physical parameters of the stack model are taken from real properties of existing technologies (MEA, bipolar plates), based on [16].

\subsection{System parameters}

Simulations are carried out with a power profile scaled from the Worldwide harmonized Light vehicles Test Cycle (WLTC-Class 3) [19]. The reference load current is computed in real time according to the desired output power and the actual stack voltage by:

$$
I_{\text {load }}=\frac{P_{W L T C}}{V_{\text {stack }}}
$$


The anode is fed with dry hydrogen and the cathode is fed with $50 \%$ air relative humidity $(\mathrm{RH})$.

The cooling temperature randomly varies according to a Gaussian normal law with $353 \mathrm{~K}$ mean and $10^{2} \mathrm{~K}^{2}$ variance. The anode and cathode pressure are always equal and the value randomly varies according to a Gaussian normal law with $2.10^{5} \mathrm{~Pa}$ mean and $\left(4.10^{4}\right)^{2} \mathrm{~Pa}^{2}$ variance. The objective is to simulate a fuel cell management system that gives dynamic reference for both temperature and pressures. No measurement accuracy is taken into account for the sensors used to correct the estimated states, the MePHYSTO-FC model providing the exact values.

The anode purge is triggered on the nitrogen stratification level given by the observer. The threshold is fixed at $5 \%$ and the purge duration is tuned to avoid purging hydrogen.

According to the expected behavior based on these parameters, the observer gains are chosen to satisfy (26) and (27) inequalities.

\subsection{State estimation}

The results presented here are computed from the states given by the observer. For the following results, the initial state values are chosen according to the expected ones at the beginning of the simulation. Some sensibility analysis has proven the robustness of the observer about initial conditions uncertainty. The relative humidities are deduced from the quantity of water in the channels with the equation:

$$
R H=100 \frac{P_{\text {vap }}}{P_{\text {sat }(T)}}=100 \frac{n_{\mathrm{H}_{2} \mathrm{O}} R T}{V P_{\text {sat }}(T)}
$$

The nitrogen buildup is expressed as a volumic saturation based on the quantity of nitrogen in the anode channel with:

$$
s_{N_{2}}=\frac{n_{N_{2} a} R T}{P_{a} V_{a}}
$$

The evolution of the relative humidities in the channels during the WLTC are represented in Figure 2. Despite the homogeneous assumption, the observer is 
able to track the average relative humidity in the channels with a good precision. There is no need to improve the relative error of the observer since it is not relevant regarding the range of relative humitidy along the channel. One should notice that the observer malfunctions with respect to the relative humidity at the cathode during the end of the cycle. This is due to the formation of liquid water that is not taken into account in the observer. The value of the resulting relative humidity is not relevant to estimate the generated liquid water. This uncertainty also rarely occurs for the relative humidity at the anode even if the Figure 2 does not illustrate it. The difference between those channels could be explained by the fact that the number of moles of water in the cathode side (state 3 ) is not directly corrected by the sliding mode. Furthermore, the channels have different dynamical equations, and in particular the water dynamics in the cathode takes into account some inlet and outlet water flows, as a function of the channel relative humidity, possibly leading to a more important uncertainty. However, it is possible based on this phenomenon to create a detection routine of liquid water for control purposes. The relative humidity can also be bounded between 0 and $100 \%$ to avoid this divergence, without a loss of information.

The estimation of the nitrogen build up is represented in Figure 3. One can notice that the observer estimation is able to capture the dynamic behavior of the average nitrogen saturation given by the $1 \mathrm{D}+1 \mathrm{D}$ model. However, as the values are small, the relative error could be important. Despite this error, it is possible with this observer to regulate the nitrogen saturation below a given level ( $5 \%$ here). The purge duration is chosen shorter than the time required to purge entirely the nitrogen from the anode. This a precaution in case of a real application as the modeling of the purge mechanism and the nitrogen build up in the anode are not well-understood. In particular, the accumulation of nitrogen at the outlet of the anode channel may not be as we model it in Figure 4, based on [20]: the stratification profile could be inaccurate, due to the model itself or the meshing. In particular, for small nitrogen saturations involved in the observer-based purge, the stratification may not occur as quickly as the purge interval ( $\sim 10 \mathrm{~s})$. A better understanding and modeling of such mechanism could 

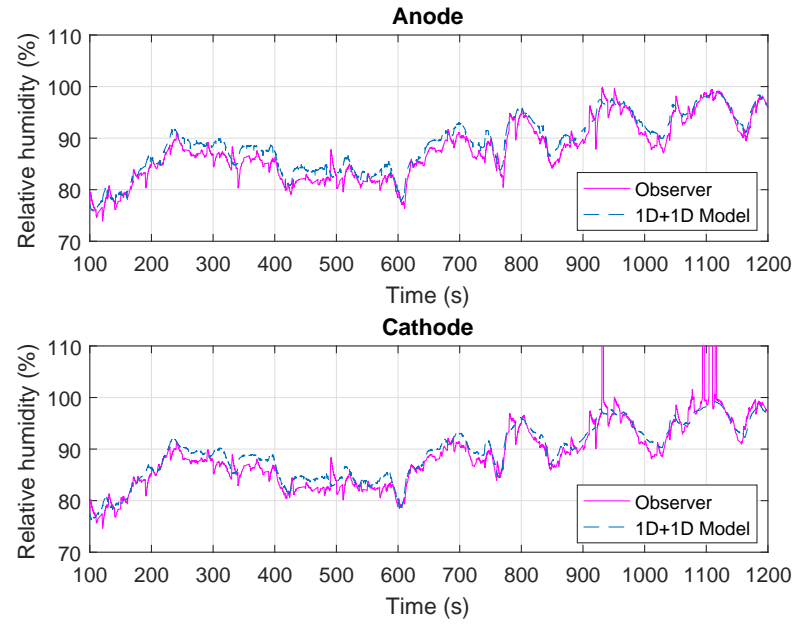

Figure 2: Evolution of the relative humidities $(\mathrm{RH})$ in the anode and cathode side. The red line represents the $\mathrm{RH}$ computed by the observer and the dash blue line the average relative humidity generated by the MePHYSTO-FC model.

lead to even better performance on anode observer-based purges. A comparison of observer-based purges and other methods can be found in [13].

\subsection{Sensitivity analysis}

Extensive simulations have been carried out to analyse the sensitivity of the estimated states to several incertainties:

- error on the input oxygen stoechiometry due to the control of the compressor;

- variation of cathode input relative humidity with the use of a passive humidifier system;

- noise measurements on membrane resistance and anode pressure.

These incertainties are modeled by a multiplicative white gaussian noise with a unit mean and the corresponding variance listed in Table 1 . These variances have been chosen according to the expected range of variations of inputs parameters and the characteristics of the real noise measurements. Only the nominal 


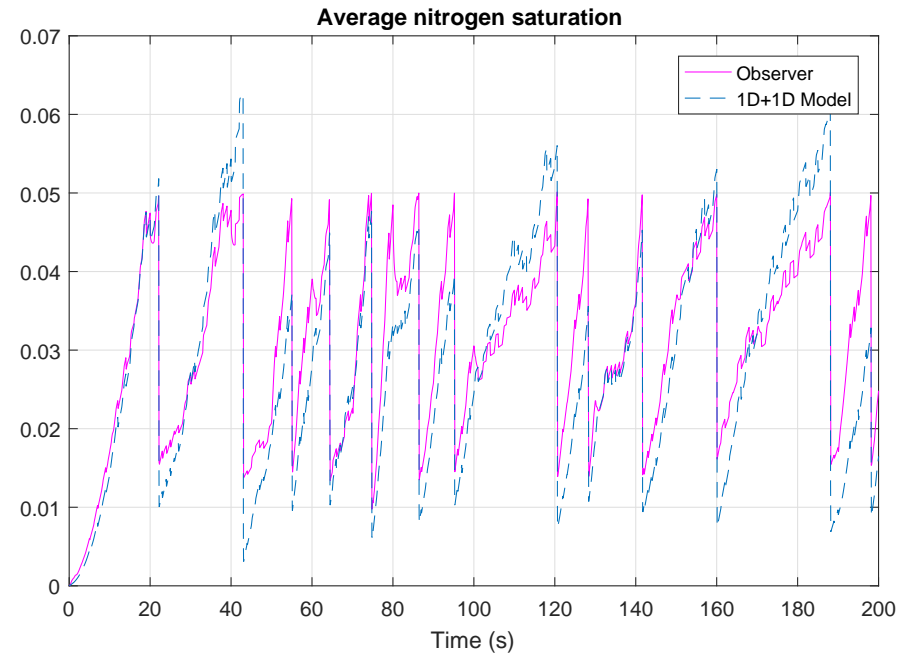

Figure 3: Estimation of the volumic saturation of nitrogen in the anode channels. The red line represents the nitrogen saturation computed by the observer and the dash blue line the average nitrogen saturation generated by the MePHYSTO-FC model.

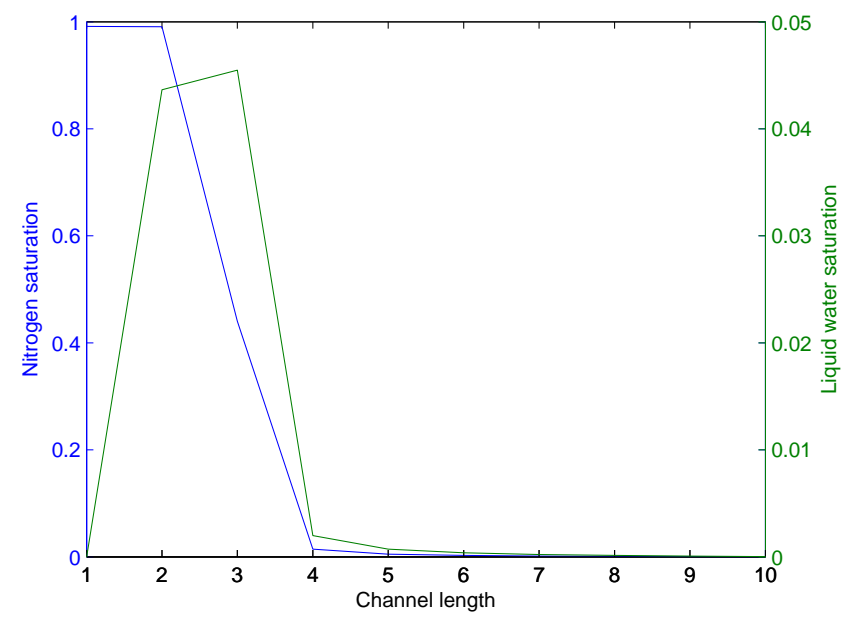

Figure 4: Stratification profile captured by the MePHYSTO-FC model. Inlet of the channel is in the $10^{\text {th }}$ mesh and outlet in the $1^{\text {st }}$. 


\begin{tabular}{|c|c|}
\hline Parameter & Variance \\
\hline \hline$S t_{O_{2}}$ & 0.5 \\
\hline$R H_{c, i n}$ & 0.5 \\
\hline$R_{m}$ & 0.1 \\
\hline$P_{a}$ & 0.05 \\
\hline
\end{tabular}

Table 1: Variance of the multiplicative noise applied to the mentioned parameters.

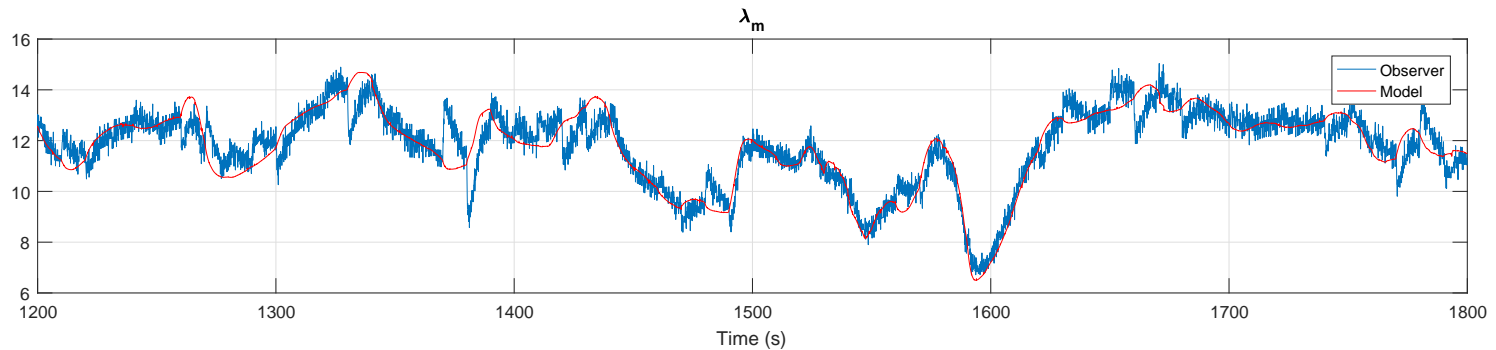

Figure 5: Evolution of $\lambda_{m}$ at the end of the WLTC. The observer estimate is affected by the measurement noise but still very close to its moving average.

value of the input oxygen stoechiometry and cathode input relative humidity are taken into account in the observer.

The observer is expected to be insensitive to the incertainties of the oxygen stoechiometry and the relative humidity due to the formulation of the sliding mode. Indeed, the gains have been determined for the whole range of operating stoechiometry and input relative humidity. Simulations validated this insensitivity for the previously mentioned incertainties during the WLTC.

Furthermore, the influence of the noise measurements can be seen in Figure 5. The convergence of the mean value is still guaranteed even if the noise affects the estimated states.

Other geometrical parameters could lead to a wrong estimation of the internal states. Indeed, the evolution of parameters caused by ageing could affect the performance of the observer. Among all parameters involved in the observer equations, the membrane thickness plays a major role, in particular in the nitrogen permeation and the diffusive flux. Despite a difference of $10 \%$ between 
the model membrane thickness and the observer one, the latter still provides the correct estimates of the internal states during a WLTC.

\section{Conclusion}

This paper present a state observer to estimate the nitrogen in the anode and the relative humidities in the channels for a PEMFC. The convergence of the algorithm has been proved using Lyapunov theory for dynamic operating conditions. Despite the homogeneous assumption, the observer is able to provide correct average estimation of the desired parameters. The presence of liquid water is not well quantified but the divergence of the observer when a flooding occur could be used as a diagnosis tool. This observer can be used in a more general control scheme, for example with a fuel cell management system, as it works for dynamic power and operating conditions. An experiment in a real test bench is planned to validate the precision of the observer.

The algorithm is protected by patent EN 1750165 .

[1] A. Kirubakaran, S. Jain, R. Nema, A review on fuel cell technologies and power electronic interface, Renewable and Sustainable Energy Reviews 13 (2009) 2430-2440.

[2] S. Specchia, Fuel processing activities at european level: A panoramic overview, International Journal of Hydrogen Energy 39 (31) (2014) 1795317968. doi:http://dx.doi.org/10.1016/j.ijhydene.2014.04.040.

[3] S. Strahl, A. Husar, J. Riera, Experimental study of hydrogen purge effects on performance and efficiency of an open-cathode proton exchange membrane fuel cell system, Journal of Power Sources 248 (2014) 474-482.

[4] T. Matsuura, J. Chen, J. Siegel, A. Stefanopoulou, Degradation phenomena in pem fuel cell with dead-ended anode, International Journal of Hydrogen Energy 38 (26) (2013) 11346-11356. 
[5] J. Chen, J. Siegel, A. Stefanopoulou, J. Waldecker, Optimization of purge cycle for dead-ended anode fuel cell operation, International Journal of Hydrogen Energy 38 (12) (2013) 5092-5105.

[6] K. Nikiforow, H. Karimaki, T. Keranen, J. Ihonen, Optimization study of purge cycle in proton exchange membrane fuel cell system, Journal of Power Sources 238 (2013) 336-344.

[7] R. Ahluwalia, X. Wang, Buildup of nitrogen in direct hydrogen polymerelectrolyte fuel cell stacks, Journal of Power Sources 171 (2007) 63-71.

[8] H. Zhao, A. F. Burke, Optimization of fuel cell system operating conditions for fuel cell vehicles, Journal of Power Sources 186 (2009) 408-416.

[9] J. Wu, Q. Liu, H. Fang, Toward the optimization of operating conditions for hydrogen polymer electrolyte fuel cells, Journal of Power Sources 156 (2) (2006) 388-399. doi:http://dx.doi.org/10.1016/j.jpowsour.2005.05.091.

[10] H. Kanani, M. Shams, M. Hasheminasab, A. Bozorgnezhad, Model development and optimization of operating conditions to maximize pemfc performance by response surface methodology, Energy Conversion and Management 93 (2015) 9-22. doi:http://dx.doi.org/10.1016/j.enconman.2014.12.093.

[11] N. Y. Steiner, D. Hissel, P. Mocoteguy, D. Candusso, Diagnosis of polymer electrolyte fuel cells failure modes (flooding and drying out) by neural networks modeling, International Journal of Hydrogen Energy 36 (4) (2011) 3067-3075.

[12] J. Luna, A. Husar, M. Serra, Nonlinear distributed parameter observer design for fuel cell systems, International Journal of Hydrogen Energy 40 (34) (2015) 11322-11332. doi:http://dx.doi.org/10.1016/j.ijhydene.2015.05.132.

[13] M. Piffard, M. Gerard, E. Bideaux, R. D. Fonseca, P. Massioni, Control by state observer of pemfc anodic purges in dead- 
end operating mode, IFAC-PapersOnLine 48 (15) (2015) 237-243. doi:http://dx.doi.org/10.1016/j.ifacol.2015.10.034.

[14] P. Schott, P. Baurens, Fuel cell operation characterization using simulation, Journal of Power Sources 156 (1) (2006) 85-91.

[15] C. Robin, M. Gerard, A. Franco, P. Schott, Multi-scale coupling between two dynamical models for PEMFC aging prediction, International Journal of Hydrogen Energy 38 (11) (2013) 4675-4688.

[16] C. Robin, M. Gerard, J. d'Arbigny, P. Schott, L. Jabbour, Y. Bultel, Development and experimental validation of a PEM fuel cell 2d-model to study heterogeneities effects along large-area cell surface, Int. J. Hydrogen Energy 40 (32) (2015) 10211-10230.

[17] K. Cooper, M. Smith, Electrical test methods for on-line fuel cell ohmic resistance measurement, Journal of Power Sources 160 (2006) 1088-1095.

[18] J. Hedrick, A. Girard, Control of nonlinear dynamic systems theory and applications, 2005.

[19] M. Tutuianu, P. Bonnel, B. Ciuffo, T. Haniu, N. Ichikawa, A. Marotta, J. Pavlovic, H. Steven, Development of the world-wide harmonized light duty test cycle (wltc) and a possible pathway for its introduction in the european legislation, Transportation Research Part D: Transport and Environment 40 (2015) 61 - 75. doi:https://doi.org/10.1016/j.trd.2015.07.011.

[20] J. Chen, J. B. Siegel, A. G. Stefanopoulou, Nitrogen blanketing front equilibria in dead end anode fuel cell operation, in: Proceedings of the 2011 American Control Conference, 2011, pp. 1524-1529. doi:10.1109/ACC.2011.5991552. 